\title{
Numerical investigation of three-dimensional separation control in an axial compressor cascade
}

\author{
Hayette Djedai ${ }^{1}$, Ramzi Mdouki ${ }^{2}$, Zakaria Mansouri ${ }^{3 *}$, Mokhtar Aouissi $^{1}$ \\ ${ }^{1}$ Laboratory of Mechanics, Amar Telidji University, Ghardaïa Road POBox 37G, Laghouat 03000, \\ Algeria \\ ${ }^{2}$ LET, Larbi Tébessi University, Constantine Road, Tébessa 12002, Algeria \\ ${ }^{3}$ ICARE CNRS, 1C, Avenue de la Recherche Scientifique, 45071 Orléans Cedex 2, France
}

Email: zakaria.mansouri@cnrs-orleans.fr

\begin{abstract}
The aerodynamic performances of three-dimensional compressor cascade are mainly influenced by secondary flow effects, such as the cross flow on the side wall and the corner separation at the wall-blade junction. Often, these secondary flows can produce a blockage and losses in the blade passage. A numerical study of active flow control using blowing technique in a linear compressor cascade has been carried out, in order to eliminate the 3D boundary layer stall and enhance the aerodynamic performance. The numerical simulation is performed using steady and incompressible RANS (Reynolds averaged Navier- Stokes) equations with Realizable k- $\varepsilon$ turbulence model. Good agreement is found between numerical results and experimental data, in particular pressure coefficient $\left(C_{P}\right)$ and total pressure loss coefficient $(\varpi)$. A detailed flow topology analysis is mentioned and gave the flow structure and the separation zone behavior. It was found that the blowing slot should be located upstream the dual nature critical point which represents the origin of separation. Furthermore, three suction slot configurations are used to control the separation, whereas one configuration on the suction side of the blade and two configurations are on the endwall. The first endwall slot is located parallel to the suction side of the blade and the second one is located perpendicular to the axial chord of the blade. Results indicate that the removal of the corner separation was not achieved using the slots on the blade suction side and on the endwall parallel to the suction side. Contrariwise, the perpendicular endwall slot was found to be most effective and the 3D separation is completely disappeared.
\end{abstract}

Keywords: Axial Compressor, Boundary Layer Blowing, Flow Control, Flow Topology, Separation.

\section{INTRODUCTION}

In recent years, reducing jet engine weight has become an extensive research area in the aerospace community. This weight reduction is resulted from the need of improving the engine's overall efficiency, which is affected by two main factors. The first on is, reducing the specific fuel consumption (SFC) and the second one is reducing the number of compressor's stages. The compressor efficiency of modern jet engines is approximately $90 \%$, in which more than 15 stages are used to provide the required total pressure rise [1]. To reduce the number of stages by keeping the same total pressure rise, it is necessary to increase the work per stage. Several methods are used to increase the work per stage including the suppression or reduction of the corner separation. This later is formed due to the heavy adverse pressure gradient, which is resulted from the interaction between the thin boundary layer of the blade and the thick one of endwall. The low-energy fluid resulted from this interaction then creates the corner vortex. Commonly used techniques to reduce the corner separation include passive and active control (e.g., vortex generators, cavities, blowing, suction and oscillatory blowing/suction). It was shown that passive flow control using vortex generators can reduce total pressure losses by affecting the corner separation at the crossing of the wall and blade [2,3]. It was reported also that active flow control using blowing, suction or synthetic jets can decrease greatly the total pressure losses by removing the corner separation [4-9].

Gbadebo et al [4] adopted boundary layer suction to control 3D corner separation in an axial compressor cascade. The authors used computational fluid dynamic (CFD) approach in this investigation with a suction rate up to $0.7 \%$ of the incoming mass flow and different slot configurations. It was found that the slot configurations used on the suction side of the blade reduce but does not remove the 3D corner separation. Moreover, the slots of the endwall (EW) which are parallel to the section side of the blade are more effective. They found that the 3D corner separation can be fully removed if the EW slot's length is $74 \%$ axial chord of the blade. Nerger et al [5] adopted experimental blowing technique in both endwall and 
suction side of highly loaded compressor stator cascade. For the baseline configuration (without control), they noticed that the flow behavior is changed to a massive boundary layer separation with high secondary flow, when the inlet flow angle is increased. Since the flow behavior changes with increasing incidence a combination of blowing at the endwall and at the suction side was used. It was found that the endwall blowing effectively reduces the corner separation and the suction side blowing reduces the boundary layer separation. In addition, this combination of blowing becomes more efficient when changing the blowing mass flow rate. Gmelin et al [6] investigated active flow control on a highly loaded subsonic compressor cascade using both numerical and experimental approaches. The active flow technique consists of three concepts of steady jets, pulsed jets, and zero mass flow jets (synthetic jets), in two different locations: at the endwall and the blade suction side. Here, the endwall slot is perpendicular to the suction side of the blade, unlike the used one in the references [4] and [5]. Several configurations are used and include different slot locations and blowing angles. It was reported that all steady and pulsed jets needed only a small mass flow rate (below $0.5 \%$ ) to be effective. For all blowing slot configurations, the best control location was slightly upstream of the boundary layer separation. Furthermore, their experimental and numerical results showed that optimal blowing slot configurations are able to reduce the total pressure loss by $13 \%$ and increase the static pressure rise by $9 \%$.

With the above review in mind and to the reader's knowledge, this contribution performs a series of numerical simulations intended to remedy the 3D corner separation in an axial compressor cascade. The investigated cascade is linear, subsonic and composed of NACA65-009 blades. Three blowing slot configurations are used, in which two at the endwall and one at the blade suction side. Moreover, steady Reynolds averaged Navier-Stokes (RANS) approach closed by the Realizable $\mathrm{k}-\varepsilon$ turbulence model is used in this investigation.

\section{CASCADE CONFIGURATION AND GRID}

The linear subsonic compressor cascade described in Table 1 is simulated in this paper. It is representative of highly loaded compressor stator blades found in modern jet engine compressors. It consists of NACA65-009 airfoils and was tested experimentally by Wei et al [10]. According to the experimental data, the inlet of the tested domain is located at 2.16 axial chords upstream of the leading edge of the blade and the outlet is at 1.36 axial chords downstream of the trailing edge. The computational domain is displayed in Figure 1 and it consists of a half blade with endwall and only one blade passage. Since steady RANS is used, the periodicity and the symmetry hypothesis can be considered.

Three structured girds were tested in order to full-fill the grid sensitivity requirements of the present study. A multiblock technique is adopted using ANSYS-Workbench R16.0 to generate the grids. For all grids, the first cell thickness normal to the blade wall is $\mathrm{y}=5 \times 10^{-6} \mathrm{~m}$ and corresponds to the dimensionless wall vector $\mathrm{y}^{+} \sim 5$. The medium grid contains 1 million cells is displayed in Figure 2, it was generated using the multi-block technique with $\mathrm{H}-\mathrm{O}-\mathrm{H}$ topology.
Table 1. Geometric parameters of the cascade

\begin{tabular}{cccc}
\hline \hline Parameter & Symbol & Magnitude & Unit \\
\hline Chord & $\mathrm{c}$ & 15 & $\mathrm{~cm}$ \\
Solidity & $\sigma$ & 0.134 & - \\
Aspect Ratio & $\mathrm{AR}$ & 1 & - \\
Stagger angle & $\lambda$ & 42.7 & $\circ$ \\
Incidence & $\mathrm{i}$ & 4 & $\circ$ \\
angle & & & \\
Reynolds & $\mathrm{Re}$ & $3.82 \times 10^{5}$ & - \\
number & $\mathrm{P}$ & \\
\hline \hline
\end{tabular}

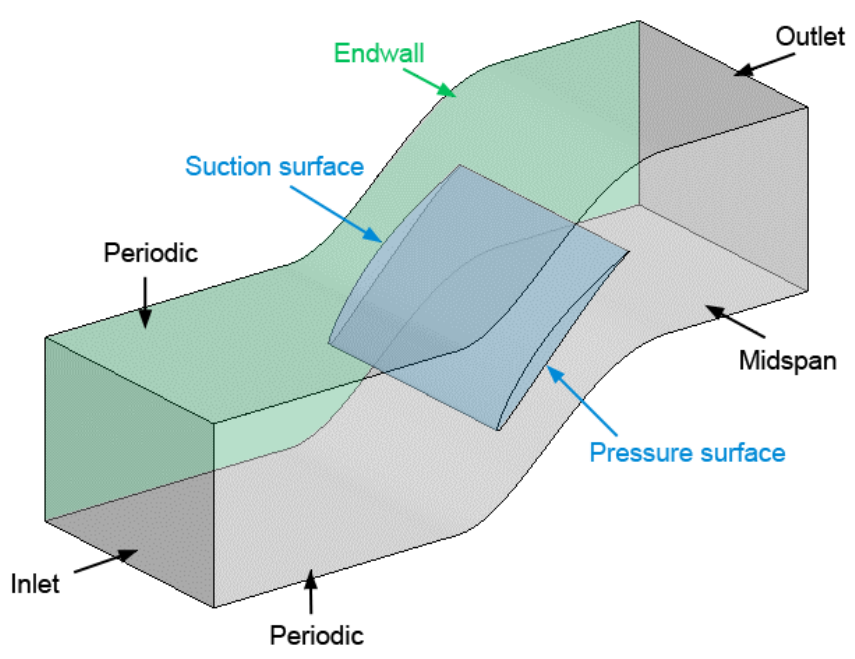

Figure 1. NACA65-009 cascade geometry with the computational domain

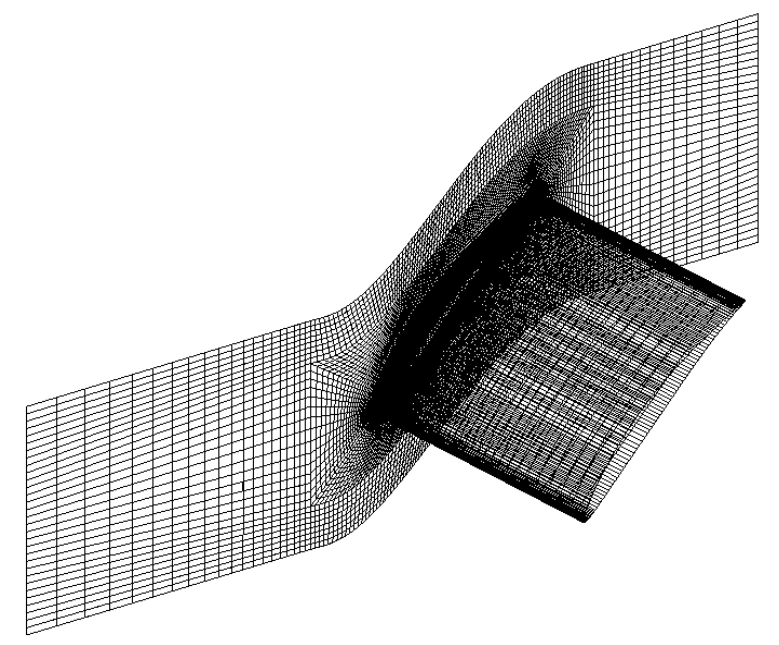

Figure 2. Three-dimensional medium grid of NACA65-009 cascade

\section{COMPUTATIONAL PROCEDURE BOUNDARY CONDITIONS}

The code used for the simulations is ANSYS-Fluent R16.0 CFD software. The finite-volume method is sued to solve the governing equations. The calculations were carried out considering the steady state, incompressible and turbulent conditions. The SIMPLE algorithm is applied for the pressurevelocity coupling. A second order upwind scheme is used for 
the momentum, turbulence kinetic energy and turbulence dissipation rate equations. All the computations were carried out till the residuals converged with criteria of $10^{-4}$ for all equations. The eddy-viscosity Realizable $\mathrm{k}-\varepsilon$ turbulence model [11] is used to close the system of Reynolds-averaged equations. The enhanced wall treatment with pressure gradient effects was chosen to model the boundary layer $[12,13]$.

The boundary conditions imposed on the computational domain are shown in Figure 1. The experimentally measured inlet velocity profile [10] is considered to define the inlet condition using User-Define Function (UDF technique) with a turbulence intensity $I=0.8 \%$. At the outlet, static pressure is specified with the extrapolating technique from neighboring interior cells. Since the flow is assumed to be periodic in the blade normal direction, periodic condition is imposed along the pitchwise boundaries. For the blade surface and the endwall, a non-slip adiabatic wall condition is specified.

\section{FLOW CONTROL STRATEGY AND SLOT ARRANGEMENTS}

The strategy of flow control in 3D cascade depends on the flow pattern (topology) at the endwall and the blade surface (particularly, the suction surface). The flow field topology consists of visualizing the critical points, such as nodes $(\mathrm{N})$, saddle points $(\mathrm{S})$ and focuses $(\mathrm{F})$ on the solid surfaces. This helps to localize the main separation line, which is mandatory to know for placing the control devices. Oil streak lines are usually used in the experiments to visualize the critical points. However, this kind of experimental data is not available for the present NACA cascade, shown in Figure 1. For this reason, numerical streamlines must be used to identify the main separation line with its corresponding points. Thus, a threedimensional simulation of the baseline cascade (without control) has been carried out. Results of the 3D flow field and flow topology are shown in figures 3 and 4 respectively.

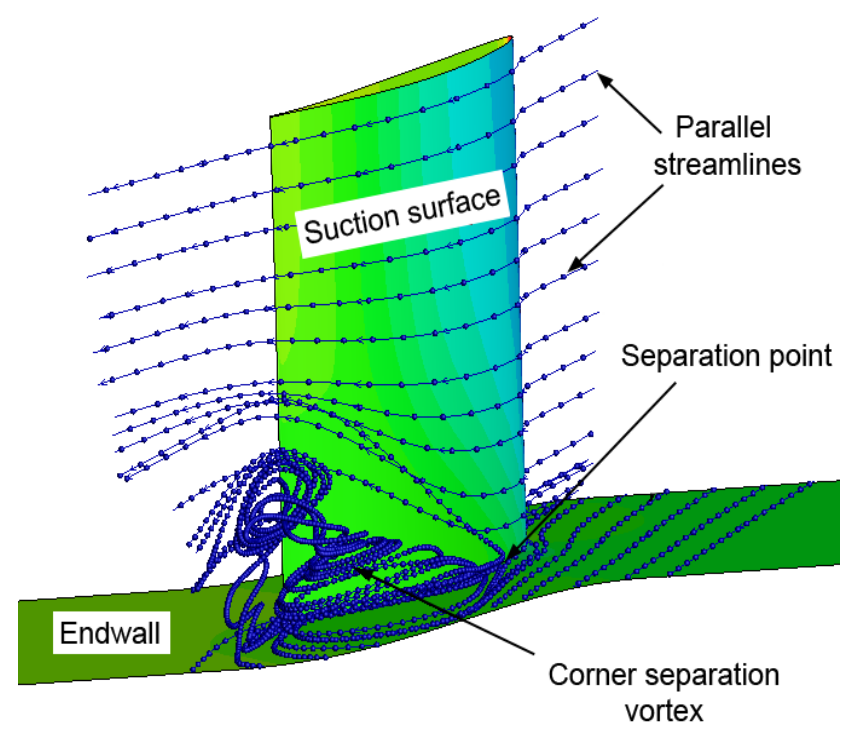

Figure 3. 3D flow field on the suction surface with the corner stall vortex.
Following the observation of the formation of the corner vortex in Figure 3, it is clear that it leads to block the cascade passage area. This is due to the reversed flow under the effect of adverse pressure gradient. The vortex system must be analyzed regarding the related critical points at the solid surfaces. From the figure 4 it can be seen that the flow revels two nodes $\left(\mathrm{N}_{1}\right.$ and $\left.\mathrm{N}_{2}\right)$, three saddle points $\left(\mathrm{S}_{1}, \mathrm{~S}_{2}\right.$ and $\left.\mathrm{S}_{3}\right)$ and one focus $\left(F_{1}\right)$. The origin of the separation line (red dashed line) is at the saddle separation point $\mathrm{S}_{1}$ and its corresponding nodal point $\mathrm{N}_{1}$. The separation line expands to the detachment node $\mathrm{N}_{2}$ with a global length of around $90 \%$ of the axial chord. Note that $\mathrm{N}_{2}, \mathrm{~S}_{2}$ are near the wall-blade junction, where $\mathrm{N}_{2}$ is at the endwall and $\mathrm{S}_{2}$ is at the suction surface, both at around $9 \% \mathrm{c}$.

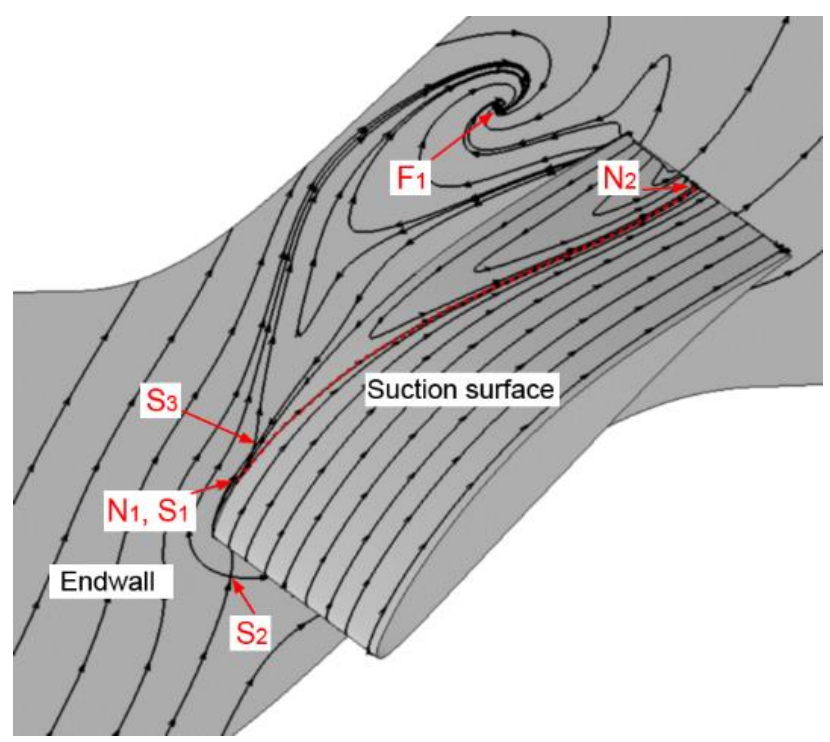

Figure 4. Predicted limiting streamlines on the blade suction surface and the endwall.

This observation gives an idea about the slot placing, which must be located on the endwall or on the suction surface at the origin point of separation of $9 \% \mathrm{c}$. Based on the above analysis, three slot arrangements are considered in this study to identify the optimum control configuration, as shown in figure 5. For all slots, the blowing ratio is fixed to $\mathrm{M}=\dot{\mathrm{m}}_{\text {jet }} / \dot{\mathrm{m}}_{\text {total }}=0.5 \%$ and the slot width at $\mathrm{w}=1 \% \mathrm{c}$. The slot arrangements are described as follows.

First, one slot positioned on the blade suction surface at about $9 \% \mathrm{c}$ and along the blade span. It is referred as to SA, as illustrated in Figure 5. The slot is rectangular and inclined by $45^{\circ}$ accordingly to the axial chord. This inclination angle leads to a better interaction between the main stream and the highenergy blowed jet in the boundary layer region along the streamwise direction. Second, two slots are positioned on the endwall at the origin of the separation line. The first one runs parallel to the suction surface from $9 \%$ to $99 \%$ and it is labeled (WA). It is purposely located along the chordwise direction, where a new tangential boundary layer must replace the separated one, which contains low-energy fluid. The second endwall slot, referred to as (WB), located perpendicular to the axial chord at $9 \% \mathrm{c}$. The slot is inclined by $45^{\circ}$ and it has a length of $18 \% \mathrm{c}$. In this configuration, the control focuses on the low-energy boundary layer of the endwall, which it would be blowed off to prevent its interaction with the suction surface. 


\section{RESULTS AND DISCUSSION}

\subsection{Grid sensitivity study}
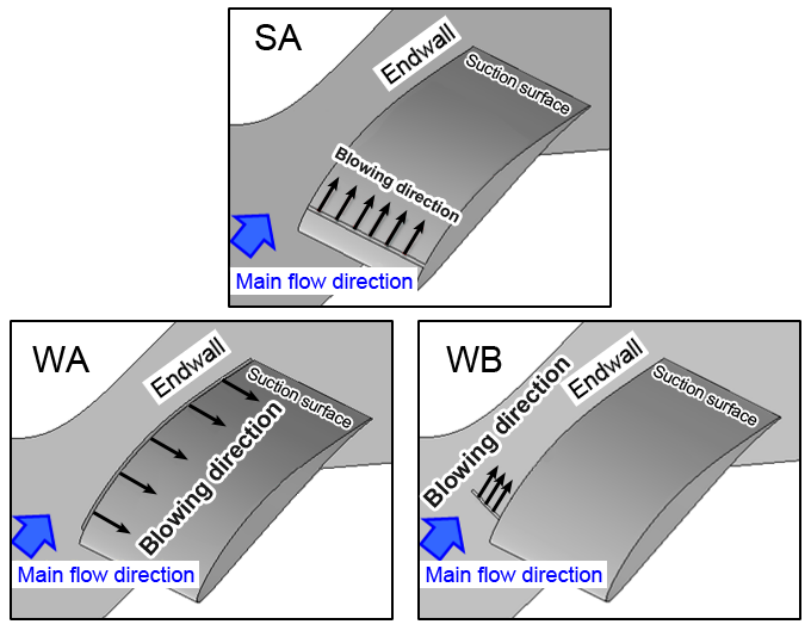

Figure 5. Schematic of blowing slots on the endwall and the blade suction surface

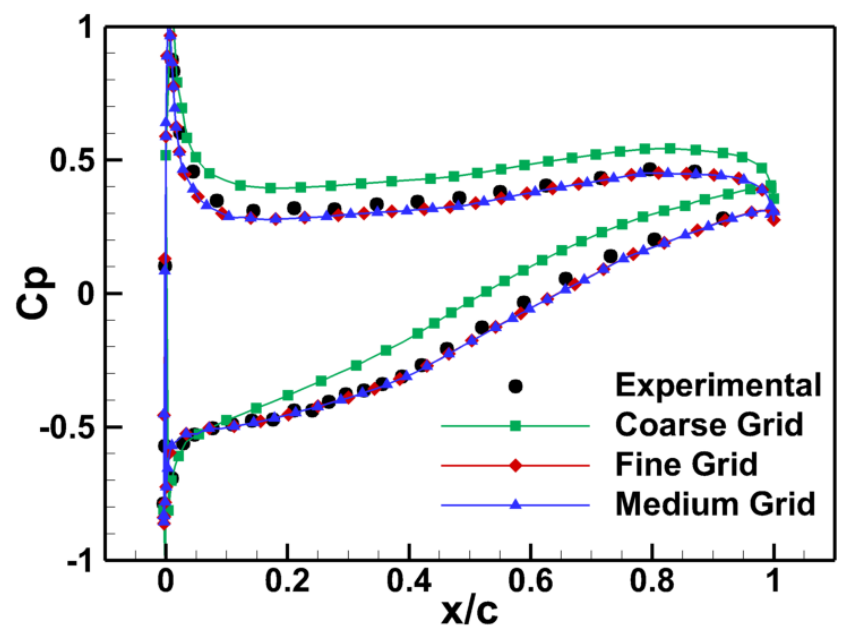

Figure 6. Grid sensitivity solutions of the static pressure coefficient $(\mathrm{Cp})$ at midspan

To ensure a sufficient modeling of the flow around the compressor cascade, a grid sensitivity analysis is conducted. Three different grids are used in this study, which are: coarse with 510150 cells, medium with 1087125 cells and fine with 1544100 cells. The influence of the grid refinement on the numerical solution, e.g., the static pressure coefficient $(\mathrm{Cp})$ at the blade surface $(\mathrm{h}=50 \%)$, is shown in Figure 6 . The results of the coarse grid show different tends compared to the experimental data as well as the results of the fine and medium grids. The coarse grid results overestimate the static pressure coefficient distribution compared to the other grids. Concerning the $\mathrm{Cp}$ results of the fine and medium grids, there are no substantial differences compared with the experiment. Since the fine grid gives the same results as the medium grid and to reduce the computational efforts, the medium grid is chosen for all calculations in the present study.

\subsection{Validation with the experimental data}

Figure 7 shows comparisons of surface static pressure coefficient $(\mathrm{Cp})$ between experimental data and present CFD results. The static pressure coefficient is defined as: $C_{p}=\left(p-p_{\infty}\right) /\left(p_{t \infty}-p_{\infty}\right)$

Two locations were chosen for the comparisons, in order to examine the capability of the turbulence model in predicting both attached and detached flow on the blade. At the midspan where $\mathrm{h}=50 \%$ (Figure 7.a), the numerical results of $\mathrm{Cp}$ are in very good agreement with the experimental data. This indicates that the model used is suitable for attached boundary layer condition. Near the endwall at $\mathrm{h}=5.4 \%$, the model shows slight discrepancies between the numerical and the experimental $\mathrm{Cp}$. Since it is an eddy-viscosity model, it stays limited in such kind of detached flows. In general, the CFD results are acceptable and the Realizable $\mathrm{k}-\varepsilon$ model well be kept to investigate the corner separation control.
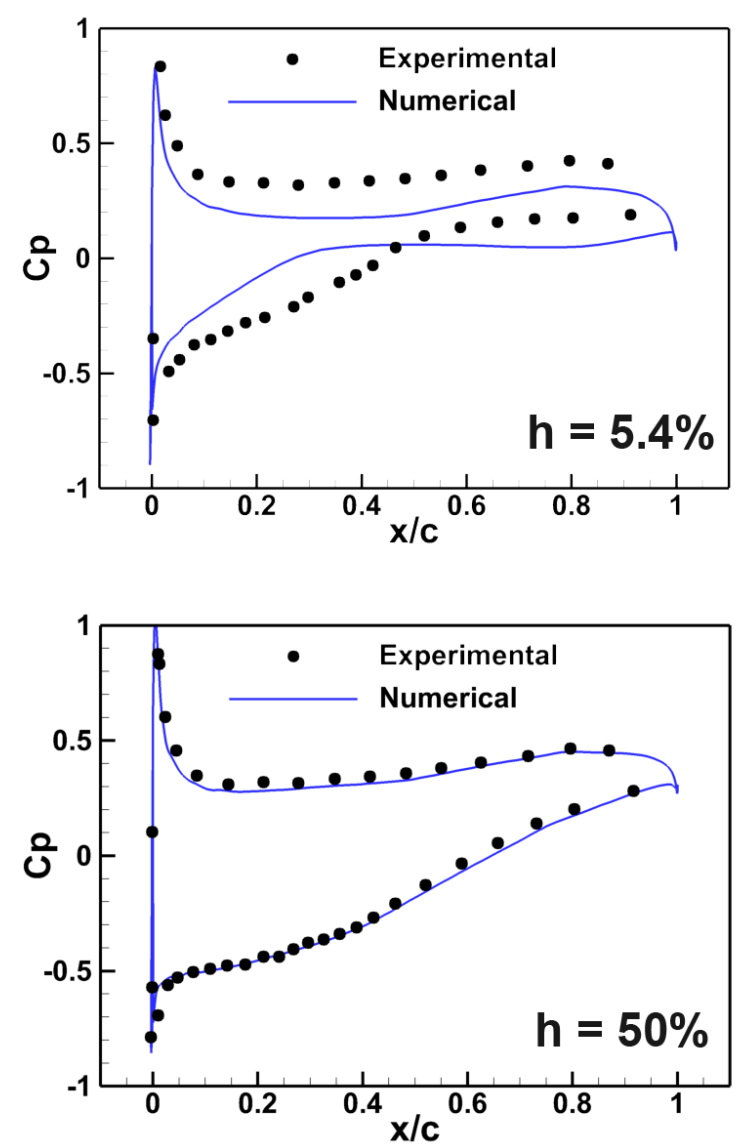

Figure 7. Comparison of $\mathrm{Cp}$ between experimental data and numerical results at $\mathrm{i}=4^{\circ}$

The figure 8 illustrates comparisons of contours of the total pressure loss coefficient, which is defined as:

$\varpi=\left(p_{t \infty}-p_{t}\right) /\left(p_{t \infty}-p_{\infty}\right)$

The contours are plotted at the cascade exit plane $(36.3 \%$ chord) downstream from the trailing edge. The total pressure loss coefficient is used in order to quantify the blockage effect of the corner separation at the cascade outlet. The blockage region is mentioned by the gradient from the green to the red 
color. It represents around $35 \%$ of the cascade outlet passage. From the figure, it can be seen that the predicted contours of $\varpi$ are in good agreement with the experiment. This confirms that the used Realizable k- $\varepsilon$ is acceptable to model such kind of flows.

\section{Experimental Numerical}
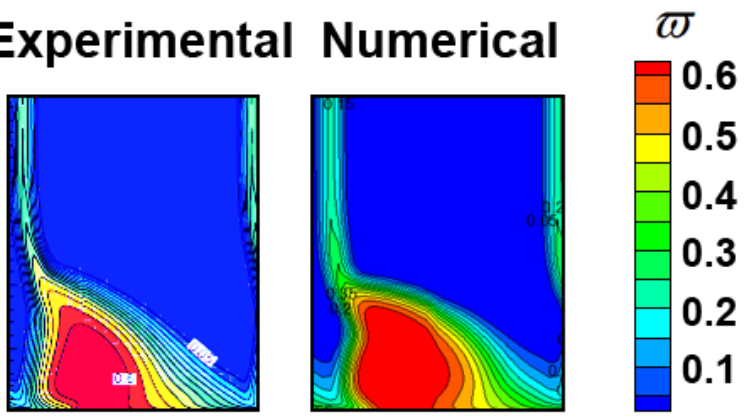

Figure 8. Comparison of exit total pressure loss coefficient between experimental data and numerical results at $\mathrm{i}=4^{\circ}$
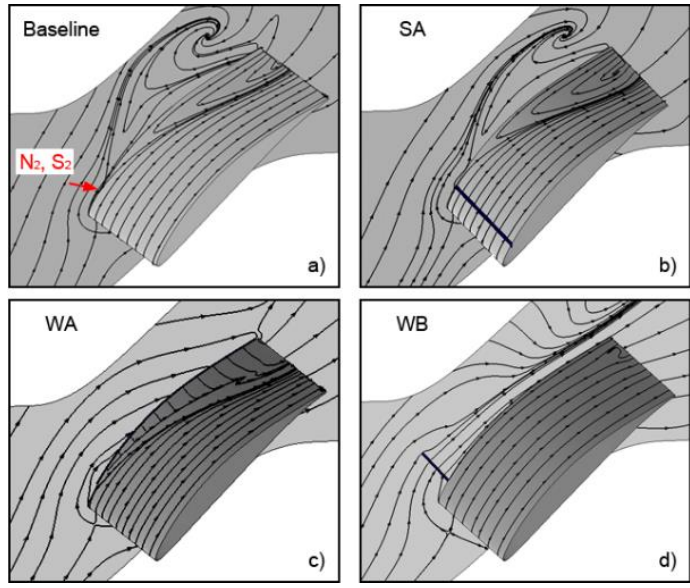

Figure 9. Predicted limiting streamlines on the suction surface and the endwall without and with blowing slot

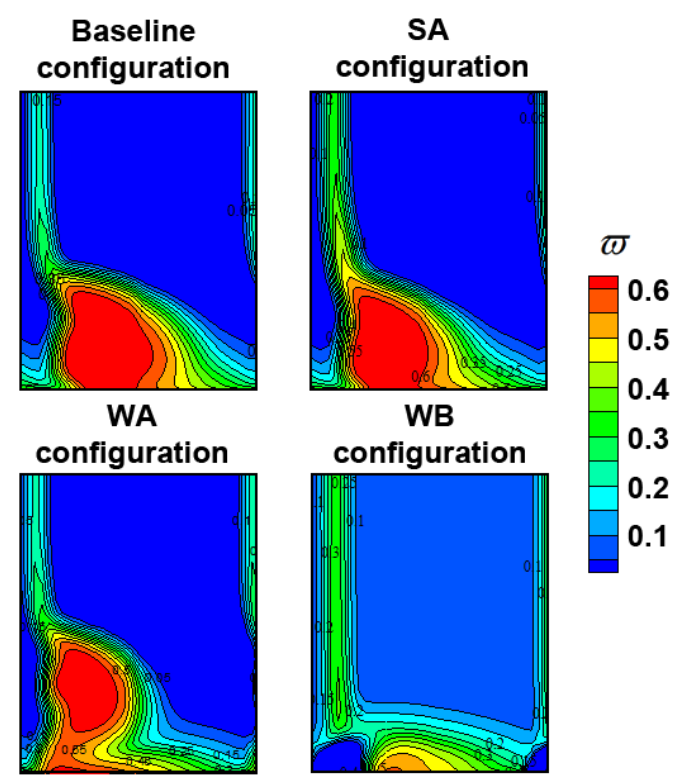

Figure 10. Comparison of exit total pressure loss coefficient between solted an unsloted configurations

\subsection{Effects of boundary layer blowing on flow topology}

Figure 9 shows the limiting streamlines on the blade suction surface and the endwall for the three slots configurations and the baseline configuration. For SA configuration (Figure 9.b), the corner separation is still present at the wall-blade junction. This is due to the slot placement a bit upstream the critical points $\mathrm{N}_{2}, \mathrm{~S}_{2}$ (the origin of the separation line). Thus, no significant changes on the 3D separation topology are found using this configuration. For the WA configuration (Figure 9.c), it can be seen that the separation on the endwall is no longer exist, since the slot has been placed parallel to the suction surface along the axial chord direction. However, the separation is not removed from the suction surface and its topology is modified compared with the baseline limiting streamlines. Further, it can be concluded that the corner separation is not provoked by the suction surface but by the endwall. For the WB configuration (Figure 9.d), it is clearly visible that the entire separated region has been totally removed. It is due to the slot arrangement, which affects and modifies the boundary layer of the endwall and consequently leads to generate only attached flow in the junction. Finally, the WB configuration proves its capability to eliminate the 3D separation among the other slots and it must be considered as the optimal choice.

\subsection{Effects of boundary layer blowing on total pressure loss}

Figure 10 presents the predicted contours of the total pressure loss coefficient at the cascade exit plane $(36.3 \%$ chord) for the three slots configurations and the baseline configuration. Note that only numerical contours are shown for the baseline configuration, since it is similar to the experiment. For SA configuration, the losses remain almost the same as the baseline configuration, because of the 3D separation which has not been removed. As mentioned before, it is due to the slot placement upstream the origin of the separation line. For WA slot, the total pressure loss is slightly reduced as a consequence of the endwall separation removal. In this case, the corner separation tends to be quasi twodimensional separation as clearly observed in Figure 9.c. Therefore, the cascade outlet passage is increased by around $5 \%$ relative to the cascade without slot. Regarding WB configuration, it is clear from the figure 10 that the loss core (contours of $\varpi>0.55$ ) caused by the interaction of the boundary layers of the endwall and the suction surface has been eliminated entirely. Further, what is remain here is the loss due to the endwall boundary layer and the mainstream wake (contours of $\varpi$ around 0.3 ). Moreover, when blowing is applied on the endwall and perpendicularly to the blade chord, the cascade outlet passage is increased by around $25 \%$ compared to the baseline cascade.

\section{CONCLUSION}

A numerical study was performed to control the threedimensional corner separation on NACA65-009 blading in axial compressor cascade, using active control steady blowing technique. The boundary layer blowing was investigated by modeling the flow using RANS computations. The blowing jets were obtained using tangential slots with fixed blowing ratios and slots widths. First, the flow topology for the clean cascade is analyzed and according to it, several critical points 
with the main separation line are identified. Based on the flow topology analysis, three slot arrangements are adopted which are; a slot positioned on the blade suction surface named (SA), a slot runs parallel to the suction surface from $9 \%$ to $99 \% \mathrm{c}$ labeled (WA) and a slot located perpendicular to the axial chord at $9 \%$ c, referred to as (WB). Second, grid sensitivity study is done for three different meshes and the medium grid of 1 million cells was chosen to perform the present investigation. Third, validations of the obtained results with the experimental data are performed for the surface static pressure coefficient $(\mathrm{Cp})$ and the total pressure loss coefficient $(\varpi)$. The used Realizable $\mathrm{k}-\varepsilon$ turbulence model showed promising results in predicting $\mathrm{Cp}$ at baled midspan, but it showed a slight discrepancy near the endwall. In addition, its capability to replicates the experimental losses $\varpi$ was adequate. Fourth, the effect of blowing on flow topology is examined for all slots configurations. Boundary layer blowing using SA configuration did not affect the 3D separation, due to the slot location upstream the origin of the separation line. The WA slot removed the limiting streamlines on the endwall and reduced the total pressure losses but did not remove the $3 \mathrm{D}$ separation. The cascade outlet passage is increased by around $5 \%$ using this configuration. Removing the 3D separation was achieved with WB configuration, where the endwall boundary layer blowed-off entirely. Further, the losses took the minimum values around $\varpi \sim 0.3$ and the cascade outlet passage increased by $25 \%$ compared with the passage of the baseline cascade. Finally, the optimum slot should be on the endwall to be sure to eliminate the limiting streamlines and is recommended to run perpendicular to the suction surface from a position at the origin of the separation line near the leading edge.

\section{REFERENCES}

[1] Lord A.K., MacMartin D.G., Tillman T.G. (2000). Flow control opportunities in gas turbine engines, AIAA Fluids 2000, Denver, CO, USA.

[2] Hergt A., Meyer R., Müller M.W. (2008). Loss reduction in compressor cascades by means of passive flow control, Proc. ASME Turbo Expo 2008, Berlin, Germany.

[3] Hergt A., Meyer R., Engel K. (2012). Effects of vortex generator application on the performance of a compressor cascade, ASME. J. Turbomach, Vol. 135, No. 2, pp. 021026-021026-10. DOI: $\underline{10.1115 / 1.4006605}$

[4] Gbadebo S.A., Cumpsty N.A., Hynes T.P. (2007). Control of three-dimensional separations in axial compressors by tailored boundary layer suction, ASME. J. Turbomach, Vol. 130, No. 1, pp. 011004-011004-8. DOI: $\underline{10.1115 / 1.2749294}$
[5] Nerger D., Saathoff H., Radespiel R., Gümmer V., Clemen C. (2011). Experimental investigation of endwall and suction side blowing in a highly loaded compressor stator cascade, ASME. J. Turbomach, Vol. 81, No. 134, pp. 021010-021010-12. DOI: $10.1115 / 1.4003254$

[6] Gmelin C., Zander V., Hecklau M., Thiele F., Nitsche W., Huppertz A., Swoboda M. (2012). Active flow control concepts on a highly loaded subsonic compressor cascade: résumé of experimental and numerical results, ASME. J. Turbomach, Vol. 134, No. 6, pp. 061021-061021-9. DOI: 10.1115/1.4006308

[7] Gümmer V., Goller M., Swoboda M. (2008). Numerical investigation of end wall boundary layer removal on highly loaded axial compressor blade rows, ASME. J. Turbomach, Vol. 130, No. 1, pp. 011015-011015-9. DOI: $10.1115 / 1.2749297$

[8] Zander V., Hecklau M., Nitsche W., Huppertz A., Swoboda M. (2011). Active flow control by means of synthetic jets on a highly loaded compressor cascade, $P$. I. Mech. Eng. A-J. Pow. Energ, Vol. 225, No. 7, pp. 897-906. DOI: $10.1177 / 0957650911410275$

[9] Zander V., Nitsche W. (2013). Control of secondary flow structures on a highly loaded compressor cascade, P.I. Mech. Eng. A-J. Pow. Energ, Vol. 227, No. 6, pp. 674-682. DOI: $10.1177 / 0957650913495538$

[10] Ma W., Ottavy X., Lu L., Leboeuf F., Gao F. (2011). Experimental study of corner stall in a linear compressor cascade, Chinese. J. Aeronaut, Vol. 24, pp. 235-242. DOI: $\underline{10.1016 / \mathrm{S} 1000-9361(11) 60028-9}$

[11] Shih T.H., Liou W.W., Shabbir A., Yang Z., Zhu J. (1995). A new $\mathrm{k}-\varepsilon$ eddy viscosity model for high Reynolds number turbulent flows, Comput. Fluids, Vol. 24, pp. 227-238. DOI: 10.1016/0045-7930(94)00032-T

[12] Chen H.C., Patel V.C. (1988). Near-Wall turbulence models for complex flows including separation, AIAA. $J$, Vol. 26, pp. 641-648. DOI: $\underline{10.2514 / 3.9948}$

[13] Kader B. (1993). Temperature and concentration profiles in fully turbulent boundary layers, Int. J. Heat. Mass. Transf, Vol. 24, pp. 1541-1544. DOI: $\underline{10.1016 / 0017-9310(81) 90220-9}$

\section{NOMENCLATURE}

$\begin{array}{ll}\mathrm{Cp} & \text { static pressure coefficient } \\ \mathrm{h} & \text { blade height } \\ \mathrm{i} & \text { incidence angle } \\ \mathrm{k} & \text { turbulente kinetic energy }\end{array}$

\section{Greek symbols}

$\varepsilon \quad$ turbulente dissipation rate $\varpi$ total pressure loss coefficient 